\title{
Patterns of synchronization in the hydrodynamic coupling of active colloids
}

\author{
Giovanni M. Cicuta, ${ }^{1,2,{ }^{*}}$ Enrico Onofri, ${ }^{1,2}$ Marco Cosentino Lagomarsino, ${ }^{3,4}$ and Pietro Cicuta ${ }^{5}$ \\ ${ }^{1}$ Dipartimento di Fisica, Università di Parma, Parco Area delle Scienze 7A, IT-43100 Parma, Italy \\ ${ }^{2}$ INFN, Gruppo Collegato di Parma, Parma, Italy \\ ${ }^{3}$ Genomic Physics Group, FRE 3214 CNRS “Microorganism Genomics,” Paris, France \\ ${ }^{4}$ University Pierre et Marie Curie, 15 rue de l' Ecole de Medecine, Paris, France \\ ${ }^{5}$ Cavendish Laboratory and Nanoscience Centre, University of Cambridge, Cambridge CB3 OHE, United Kingdom
}

(Received 11 October 2011; published 10 January 2012)

\begin{abstract}
A system of active colloidal particles driven by harmonic potentials to oscillate about the vertices of a regular polygon, with hydrodynamic coupling between all particles, is described by a piecewise linear model which exhibits various patterns of synchronization. Analytical solutions are obtained for this class of dynamical systems. Depending only on the number of particles, the synchronization occurs into states in which nearest neighbors oscillate in in-phase, antiphase, or phase-locked (time-shifted) trajectories.
\end{abstract}

DOI: 10.1103/PhysRevE.85.016203

PACS number(s): 05.45.Xt, 82.70.Dd, 87.80.Cc, 05.60.Cd

\section{INTRODUCTION}

In passive colloidal particle systems, hydrodynamic interactions determine the dissipative and dynamical behavior, rather than the real-space conformation; as such they influence the response properties rather than the equilibrium behavior. However in actively driven systems that settle into a steady state, it can be the hydrodynamic interactions that determine the characteristics of the steady state. For example, in biological flows actuated by cilia [1], the hydrodynamic coupling is possibly the most important feature involved in the synchronization of cilia beats [2].

In this paper we analyze theoretically a very simple dynamical system which was recently proven to adequately describe the motion of a system of colloidal spheres, maintained in oscillations by optical traps [3,4]. Motion occurs at low Reynolds numbers [5], and hydrodynamic forces between particles depend on the relative velocity [6,7].

The remarkable feature of the model is that the hydrodynamic interaction of the spheres leads to the synchronization of their phases, for any number of beads, for all initial conditions and for generic values of physical parameters (sphere size, viscosity of the fluid, stiffness of the forces). The synchronized state depends on the number of spheres and on whether the optical traps provide attractive or repulsive forces. This produces a rich pattern of synchronized configurations.

The system of equations of motion is piecewise linear. It has periodic solutions, which are linear combinations of the normal modes. Whilst we do not have a mathematical proof which would explain why this dynamical system always converges to a synchronized solution, we have performed a normal mode analysis and we show that based on this it is possible to predict which synchronized solution is dominant. This analytical argument is in agreement with extensive numerical integration of the deterministic (no thermal noise) system and with the experiments and simulations reported in the companion paper which include the effect of thermal noise [4].

Let us outline the physical system and its model, leading to the dynamic deterministic system of equation of motion

\footnotetext{
*giovanni.cicuta@ fis.unipr.it
}

which will be our main concern in the next section. We refer the reader to the literature which details a series of experiments and their analysis. By focused laser beams, it was possible to confine effectively an array of micron-sized beads, each one in a harmonic well. These colloidal particles interact exclusively through the hydrodynamic coupling. The collective fluctuation modes resulting from hydrodynamic interactions have been studied in linear arrays [8] and ring arrays $[9,10]$. The "geometric switch" active driving model we study here was proposed in the context of cilia by the authors of Ref. [1]; it was studied analytically for two beads and for infinite linear chains by Cosentino Lagomarsino and Bassetti in Ref. [11], where they showed that an infinite linear chain of oscillators can sustain a traveling wave solution. This model was then realized experimentally in Ref. [3] by using optical tweezers to maintain a pair of colloidal spheres in oscillation by switching the positions of optical traps when a sphere reached its limit position. This rule leads to oscillations that are bounded in amplitude but free in phase and period. The interaction between the oscillators is only through the hydrodynamic flow induced by their motion; the colloidal particles in the experiment are subject to Brownian thermal fluctuations as would also be found in a biological context. This system of two spheres in harmonic traps leads to synchronized motion in antiphase [3]. A companion paper to this one reports experimental results and the effect of noise in a system of actively moving traps for many particles [4]. Very recently, Wollin and Stark [12] reconsidered this model for the case of a chain of driven oscillators, performing numerical simulations showing that for various potentials and forms of coupling the dynamic system evolves to a synchronized state which may be interpreted as a (discretized) wave propagating through the chain. The form of the wave depends on the driving forces and their being attractive or repulsive. This chain is closely related to the array of driven oscillators studied in this paper and experimentally observed [4]. The main differences are the geometry (our oscillators are tangential to a ring) and the symmetry (in our model, the driving force acts in the same way in the two phases of each cycle, i.e., as each oscillator moves forward or backward).

The periodic solutions analytically obtained in this paper may be interpreted as a wave propagating along the border 
of the ring, clockwise or counterclockwise. Indeed if $x_{j}(t)$ describes the motion of the $j$ th oscillator, our periodic solutions have the form $x_{j+1}(t+\Delta)=x_{j}(t)$, where $\Delta$ is a fixed, positive or negative, time interval, independent of $j$. In a recent paper [13] Uchida and Golestanian derived generic conditions on the driven oscillators to synchronize due to the hydrodynamic interactions. However, as it has already been noted in Ref. [12], it is not clear if such conditions would apply to the geometric switch model.

The paper is organized as follows. Section II outlines the model and the associated dynamical system. Section III shows the normal mode analysis that allows (in principle) a completely analytical determination of the periodic solutions of the system for any number of particles. Section IV describes the periodic solutions, if the number of particles is 3, 4, or 5, and the dominant solution for any number of particles. In Sec. V we replace the attractive forces with repulsive ones and obtain periodic solutions trivially related to the previous ones.
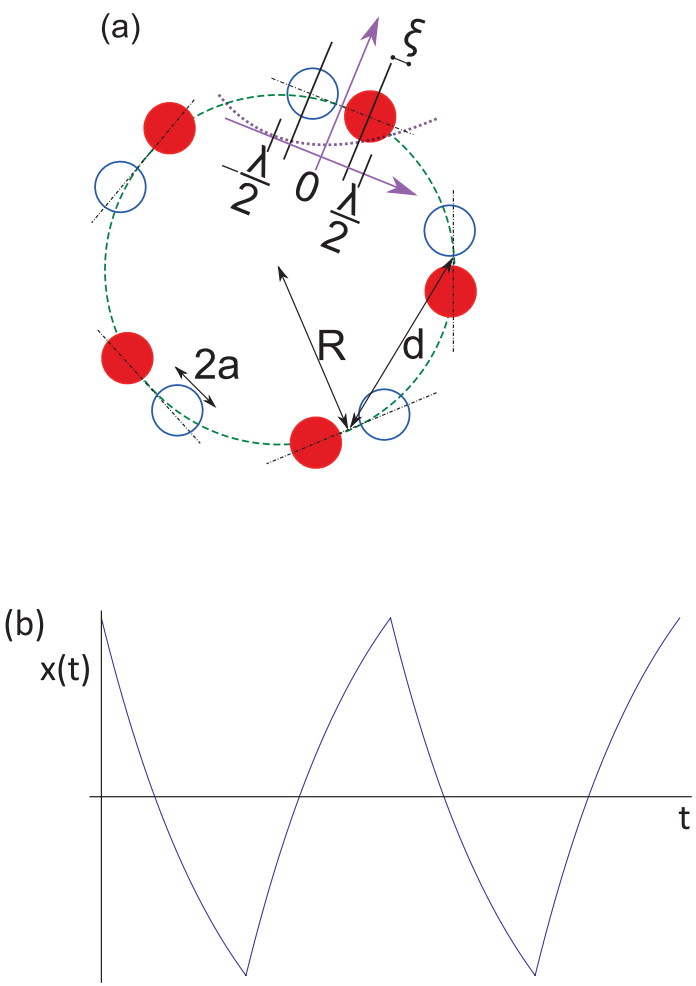

FIG. 1. (Color online) (a) Diagram of the ring arrangement of driven oscillators (here $n=5$ ), illustrating the notation used in the main text. Colloidal particles move tangential to the ring, with the center of oscillations being the vertices of a regular $n$ polygon. The local tangential coordinate system is shown for one of the top particles. The particle configuration (solid disks) corresponds to an in-phase condition, when all $n$ particles have just reached the geometric switch boundary. The particles are driven by harmonic traps toward the minimum of the potential, but they will reach first the other geometric switch boundary, when at the position indicated with open disks. This work also considers harmonic potentials with negative stiffness (i.e., repulsive) in Sec. V. (b) Trajectory of a driven oscillator with attractive driving force, uncoupled to other oscillators; $x(t)$ is measured along the tangential direction.

\section{THE MODEL}

Let us first describe the system in the absence of hydrodynamic coupling between particles. Figure 1(a) shows a ring of radius $R$, with $n$ spheres depicted at the vertices of a regular $n$ polygon. Each vertex is the center of small oscillations of a particle bound to the ring. The $j$ th particle performs oscillations about the position $R_{j}=R \theta_{j}^{(0)}=R \frac{2 \pi}{n}(j-1)$, with $j=1,2, \ldots, n$. On each side of this position, the amplitude of the oscillation is $\lambda / 2-\xi$. This is assumed to be so small that we may replace the path on the arc with a segment of straight line, tangent to the ring, and then consider the motion of the $j$ th particle on the tangent with a variable $x_{j}:-(\lambda / 2-$ $\xi) \leqslant x_{j}(t) \leqslant \lambda / 2-\xi$. The oscillations are driven by an attractive harmonic potential (or repulsive harmonic potential, as discussed later in Sec. V) with center at $s= \pm \lambda / 2$. In the hydrodynamic regime where the Reynolds number is much smaller than unity, the inertial term may be neglected.

An oscillation is set up that has a fixed amplitude, but free phase and period. This is realized through a geometric switch condition. First the trap is set at $\lambda / 2$, and the particle moves along the positive $x$ axis according to the differential equation

$$
\gamma_{0} \dot{x}(t)+\kappa\left(x(t)-\frac{\lambda}{2}\right)-f(x)=0,
$$

where $\kappa$ is the stiffness of the harmonic force, $\tau_{0}=\gamma_{0} / \kappa$ is the relaxation time, and $f$ is a stochastic force due to thermal agitation of the fluid, which is neglected in this work. $\gamma_{0}=$ $6 \pi \eta a$ is the drag on a particle of radius $a$ in the liquid of viscosity $\eta$. As the particle reaches the position $\frac{\lambda}{2}-\xi$, the attractive harmonic potential is moved at the position $-\frac{\lambda}{2}$. The particle inverts its overdamped motion until it reaches the position $-\frac{\lambda}{2}+\xi$. At this time, the potential jumps again. The resulting oscillatory motion (this is either a single bead or a bead in a system with no coupling), neglecting any stochastic force, is depicted in Fig. 1(b). The period $T_{0}$ of these uncoupled oscillations is

$$
\frac{T_{0}}{2 \tau_{0}}=\log \frac{\lambda-\xi}{\xi} .
$$

In a system of $n$ beads, at any given time, the set of positions of the particles is an $n$th dimensional vector $\vec{x}(t)=$ $\left\{x_{1}(t), x_{2}(t), \ldots, x_{n}(t)\right\}$. In the absence of the hydrodynamic coupling, each particle would perform the same periodic motion described above, a set of periodic overdamped relaxations. For any pair of particles, the "phase differences" $x_{j}(t)-x_{k}(t)$ would be set by the initial conditions (typically some random values) and be constant in time.

Let us now include the hydrodynamic interaction. The motion of the $j$ th particle originates a force on the $i$ th particle, $\vec{f}_{\alpha}^{i, j}=\left(H^{-1}\right)_{i, j}^{\alpha, \beta} v_{\beta}^{(j)}$, where $H$ is the Oseen tensor which is derived from a pairwise description and hence is adequate in the far field [5]. In agreement with previous analysis [8-10] we are led to the following system of equations:

$$
\begin{gathered}
\vec{F}_{i}-\sum_{j=1}^{n} H_{i, j}^{-1} \frac{d \vec{r}_{j}(t)}{d t}+\vec{f}_{i}(t)=0, \quad i=1,2 \ldots, n \\
\vec{r}_{i}(t) \cdot \vec{t}\left(\vec{r}_{i}\right)=0, \quad \vec{t}(\theta)=\left(\begin{array}{c}
-\sin \theta \\
\cos \theta
\end{array}\right) .
\end{gathered}
$$


The deterministic force $\vec{F}_{i}$ acting on the $i$ th particle is a harmonic force, tangent to the ring, with the geometric switch rule,

$$
\vec{F}_{i}=-\kappa\left[x_{i}(t) \mp \frac{\lambda}{2}\right] \vec{t}\left(\frac{2 \pi(i-1)}{n}\right),
$$

where $\pm \frac{\lambda}{2}$ is the coordinate of the bottom of the harmonic well.

The stochastic force $f_{i}(t)$ in Eq. (1) represents the thermal noise on the $i$ th particle, and it can be assumed that $\left\langle f_{i}(t)\right\rangle=0$, $\left\langle f_{i}\left(t_{1}\right) f_{j}\left(t_{2}\right)\right\rangle=2(\beta)^{-1}\left(H^{-1}\right)_{i j} \delta\left(t_{1}-t_{2}\right), \quad$ and $\beta^{-1}=k_{B} T$ [8]. $\vec{t}\left(\vec{r}_{i}\right)$ is a versor tangent to the ring, at the position $\vec{r}_{i}$, with counterclockwise direction. For small oscillations, the Oseen tensor can be approximated by inserting the fixed distances among centers of oscillations, then

$$
\gamma_{0} H_{i j}^{\alpha \beta}=\delta_{i j} \delta_{\alpha \beta}+\left(1-\delta_{i j}\right) \frac{3 a}{4 r_{i j}}\left(\delta_{\alpha \beta}+\frac{r_{i j}^{\alpha} r_{i j}^{\beta}}{r_{i j}^{2}}\right),
$$

where

$$
\left(\begin{array}{l}
r_{j x} \\
r_{j y}
\end{array}\right) \simeq\left(\begin{array}{l}
R_{j x} \\
R_{j y}
\end{array}\right)=R\left(\begin{array}{c}
\cos (j-1) 2 \pi / n \\
\sin (j-1) 2 \pi / n
\end{array}\right),
$$

with $r_{i j}^{\alpha} \simeq R_{j}^{\alpha}-R_{i}^{\alpha}=-r_{j i}^{\alpha}$ and $\alpha=1$ or 2 .

We project the equations of the linearized Langevin-Oseen system along the tangents, replace the global coordinates of the $j$ th particle $\vec{r}^{(j)}$ with the local one-dimensional coordinate $x^{(j)}$, and neglect the stochastic force to obtain the deterministic linear system which holds in the time interval where no particle hits its left or right boundary $\pm\left(\frac{\lambda}{2}-\xi\right)$ :

$$
\left(I+\frac{3 a}{8 R} C_{n}\right)[\vec{x}(t)-\vec{s}]+\tau_{0} \frac{d}{d t} \vec{x}(t)=0,
$$

where $\vec{x}(t)$ is the $n$-component vector of the (local) position of the particles, $\vec{s}$ is the $n$-component vector of the (local) positions of the minima of potential proper for such a time interval (i.e., $\vec{s}$ depends on the $\vec{x}(t)$ and on the previous history of the system), and $C_{n}$ is a real symmetric circulant matrix of order $n$. The first row of $C_{n}$ (which defines the entire matrix) is

$$
\begin{aligned}
C_{n}= & \left(0, \frac{\cos 2 \pi / n+\cos ^{2} \pi / n}{\sin \pi / n}, \frac{\cos 4 \pi / n+\cos ^{2} 2 \pi / n}{\sin 2 \pi / n},\right. \\
& \left.\ldots, \frac{\cos 2(n-1) \pi / n+\cos ^{2}(n-1) \pi / n}{\sin (n-1) \pi / n}\right) .
\end{aligned}
$$

\section{THE DETERMINISTIC DYNAMICS}

We now study the deterministic system described by Eq. (2). The hydrodynamic coupling affects the overdamped oscillations and determines the "phase differences" between particles. Most remarkably, it leads to collective synchronized motions, which as we will now show are best understood by considering the eigenstates of the Oseen coupling matrix $C_{n}$.

Let us call $\lambda_{j}, j=1, \ldots, n$, the eigenvalues of the matrix $C_{n}$ arranged in increasing values and $\vec{e}^{(j)}$ the corresponding normalized eigenvectors, $C_{n} \vec{e}^{(j)}=\lambda_{j} \vec{e}^{(j)}$. Let us call $h_{j}(t)=\left[\vec{e}^{(j)}, \vec{x}(t)\right]$ the projection of the position vector on the eigenvectors, that is, the normal modes of the linear system.

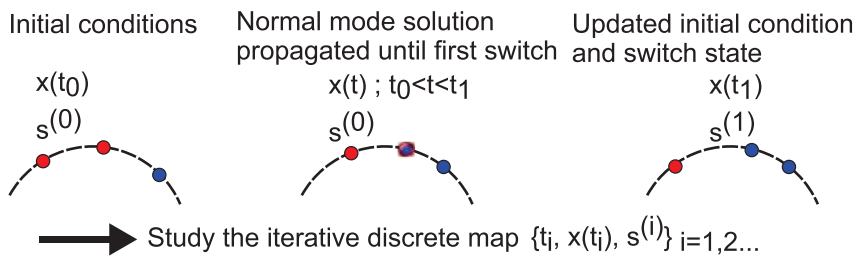

FIG. 2. (Color online) A solution of the dynamical system can be obtained by iterating the linear evolution of the system, which is simple in each of the eigenmodes, up to when any one of the beads reaches a switch position. A periodic solution for a system of $n$ particles will return to the initial configuration (both particle and trap positions) after $2 n$ switches.

Projecting the system of Eq. (2) onto each of the eigenvectors gives

$$
\vec{e}^{(j)} \cdot\left[\left(I+\frac{3 a}{8 R} C_{n}\right)(\vec{x}(t)-\vec{s})+\tau_{0} \frac{d}{d t} \vec{x}(t)\right]=0,
$$

and one easily obtains the uncoupled equations of motion for each normal mode:

$$
h_{j}(t)-\left(\vec{e}^{(j)} \cdot \vec{s}\right)+\tau_{j} \frac{d}{d t} h_{j}(t)=0, \quad \text { with } \tau_{j}=\frac{\tau_{0}}{1+\frac{3 a}{8 R} \lambda_{j}} .
$$

One sees that each normal mode $h_{j}(t)$ is related to a single exponential relaxation time $\tau_{j}$, and its linear differential equation is homogeneous, if and only if the term $\left(\vec{e}^{(j)} \cdot \vec{s}\right)$ vanishes. In general, this is not the case.

A solution of the dynamical system can be obtained with the following simple strategy (Fig. 2): Given initial positions $\vec{x}\left(t_{0}\right)$ and initial configuration of potential $\vec{s}^{(0)}$, the evolution is trivially evaluated up to the time $t_{1}$ of the first hit. At this time one entry of the vector $\vec{s}$ changes sign, and the uncoupled system of Eq. (5) is again evaluated with the new constant vector $\vec{s}^{(1)}$ up to the hit at time $t_{2}$. A basic role is then played by the continuity of each mode at the time of the hits: the final value before the hit becomes the new initial condition.

The rotational discrete symmetry of the problem allows a detailed analytic study of the coupling matrix $C_{n}$. The most relevant features are the following.

(1) For every $n$ being an even integer, the lowest eigenvalue $\lambda_{1}$ is a singlet and its corresponding eigenvector is $\vec{e}^{(1)}=$ $\frac{1}{\sqrt{n}}(1,-1,1, \ldots,-1)$.

(2) For every $n$ being an odd integer $n \geqslant 5$, the lowest eigenvalue $\lambda_{1}$ is a doublet. The two eigenvectors may be written as

$$
\begin{aligned}
\vec{e}^{(1)}= & \frac{1}{\sqrt{n}}(1, \cos (n-1) \pi / n, \quad \cos 2(n-1) \pi / n, \\
& \left.\ldots \cos (n-1)^{2} \pi / n\right), \\
\vec{e}^{(2)}= & \frac{1}{\sqrt{n}}(0, \sin (n-1) \pi / n, \quad \sin 2(n-1) \pi / n, \\
& \left.\ldots \sin (n-1)^{2} \pi / n\right) .
\end{aligned}
$$

(3) For every $n$, the matrix $C_{n}$ has the eigenvector $\vec{e}=$ $\frac{1}{\sqrt{n}}(1,1,1, \ldots, 1)$. For $n=3$ only, this constant eigenvector corresponds to the lowest eigenvalue $\lambda_{1}$. 
(4) Since $\operatorname{tr}\left[C_{n}\right]=0$, the lowest eigenvalue $\lambda_{1}<0$, and therefore the relaxation time $\tau_{1}>\tau_{0}$ for every $n$.

\section{THE ANALYTIC ASYMPTOTIC SOLUTIONS}

The dynamics of the system depends on three combinations of parameters: $\tau_{0}=\gamma_{0} / \kappa$ sets the unit of time for the evolution between hits; $3 a /(8 R)$ measures the strength of the hydrodynamic interaction and is limited by the bound $d=2 R \sin \pi / n \gg 2 a$; finally $\xi / \lambda$, with $0<\xi / \lambda<1 / 2$, fixes the amplitude of the oscillations. The periodic solutions are here obtained for arbitrary values of the parameters (within the physical bounds given above). The plots for three and four particles, in Figs. 3, 5, and 10 , have $\xi / \lambda=1 / 4$ and $3 a /(8 R)=\sqrt{3} / 5$. The plots for five particles, in Figs. 7 and 8 have $\xi / \lambda=1 / 4$ and $3 a /(8 R)=0.2$. Note that these values of $3 a /(8 R)$ are much greater than sensible experimental values $[3,4]$ and in particular are not in the far field, but they are chosen to be large here for purely graphical reasons to spread
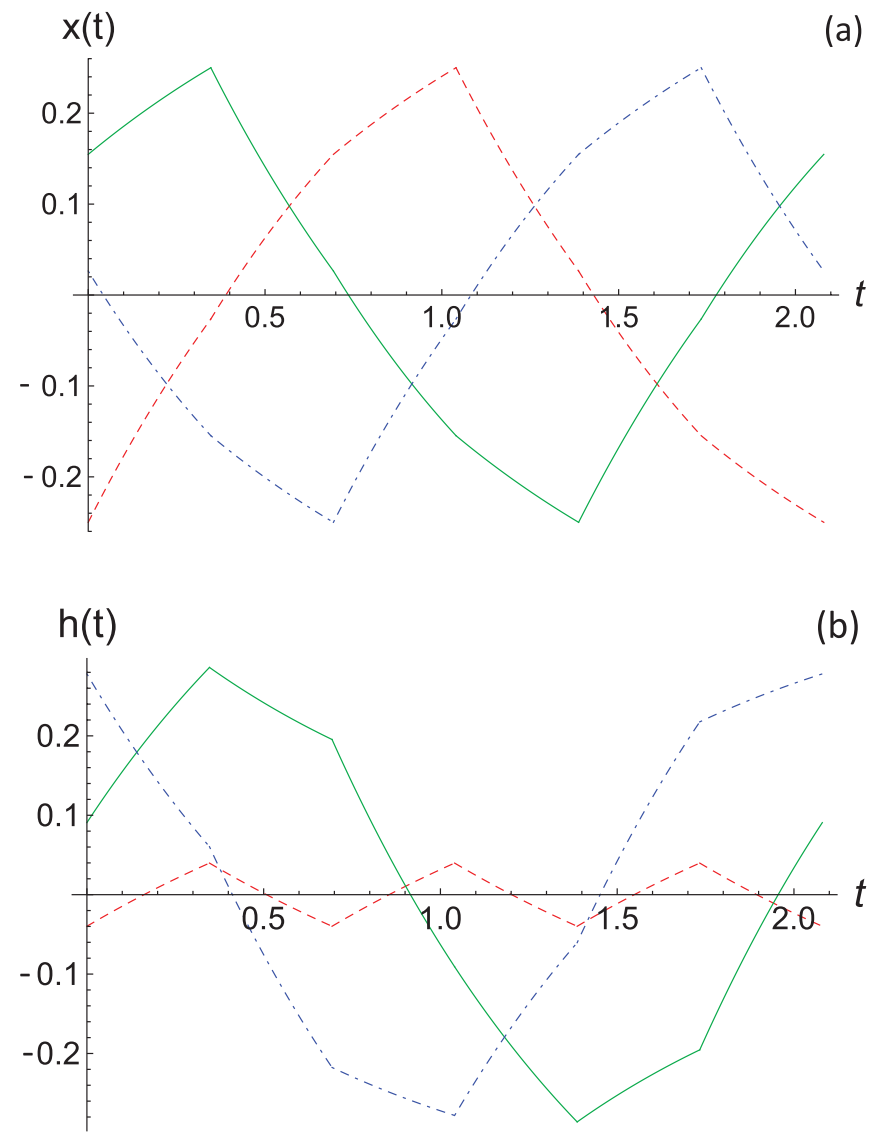

FIG. 3. (Color online) The fastest (shortest period) solution for $n=3$ is phase-locked. (a) The trajectories of particles 1 (dashed red line), 2 (solid green line), and 3 (dash-dotted blue line) are plotted versus time, for one period. (b) The normal modes $h_{1}(t)$ in red (dashed line), $h_{2}(t)$ in green (solid line), and $h_{3}(t)$ in blue (dash-dotted line) are plotted versus time. Time is expressed in units of $\tau_{0}$. This solution is not stable, as can be shown by numerical simulation, see Sec. IV E. The other solution for $n=3$ is the motion of all particles relaxing and switching in phase: it has a longer period than the one illustrated here. the relaxation times $\tau_{j}$, emphasizing the differences between periodic solutions.

\section{A. Case of $n=3$}

For $n=3$ the lowest eigenvalue of the coupling matrix $C_{3}$ is $\lambda_{1}=-\frac{1}{\sqrt{3}}$, a singlet. The next eigenvalue is a doublet, $\lambda_{2}=$ $\lambda_{3}=\frac{1}{2 \sqrt{3}}$. The corresponding eigenvectors may be written as

$$
\begin{aligned}
& \vec{e}^{(1)}=\frac{1}{\sqrt{3}}\left(\begin{array}{l}
1 \\
1 \\
1
\end{array}\right), \quad \vec{e}^{(2)}=\frac{1}{\sqrt{2}}\left(\begin{array}{c}
0 \\
1 \\
-1
\end{array}\right), \\
& \vec{e}^{(3)}=\frac{1}{\sqrt{6}}\left(\begin{array}{c}
-2 \\
1 \\
1
\end{array}\right) .
\end{aligned}
$$

We find the following periodic solutions for the system, here listed in order of decreasing duration of the periods:

(a) the three particles are synchronized in-phase;

(b) a pair of equivalent solutions, where the trajectories are phase locked (more precisely, time shifted).

Let us consider first case (a), with the configuration of particles in-phase, i.e., such that $x_{1}(t)=x_{2}(t)=x_{3}(t)$. The vector of the positions of the potential is

$$
\vec{s}= \pm \frac{\lambda}{2}\left(\begin{array}{l}
1 \\
1 \\
1
\end{array}\right)
$$

The lowest normal mode is $h_{1}(t)=\frac{1}{\sqrt{3}}\left[x_{1}(t)+x_{2}(t)+x_{3}(t)\right]$ $\rightarrow \sqrt{3} x_{1}(t)$ and it solves the equation

$$
h_{1}(t)-\left(\vec{e}^{(1)} \cdot \vec{s}\right)+\tau_{1} \frac{d}{d t} h_{1}(t)=0,
$$

where

$$
\left(\vec{e}^{(1)} \cdot \vec{s}\right)= \pm \sqrt{3} \frac{\lambda}{2} \quad \text { and } \quad \tau_{1}=\tau_{0}\left(1-\frac{\sqrt{3} a}{8 R}\right)^{-1} .
$$

Since $\left(\vec{e}^{(j)} \cdot \vec{s}\right)=0$ for $j \neq 1$, the modes $h_{2}(t)$ and $h_{3}(t)$ solve a homogeneous equation and the choice $h_{2}(t)=h_{3}(t)=$ 0 is consistent. As the three particles hit simultaneously a geometric switch border, all of $\vec{s} \rightarrow-\vec{s}$. Therefore by continuity one obtains the opposite motion in the following time interval. The three particles perform in-phase oscillations, satisfying the same equation as if they were uncoupled, as depicted in Fig. 1(b), but with the longer period

$$
\frac{T_{1}}{2 \tau_{1}}=\log \frac{\lambda-\xi}{\xi} .
$$

Let us now obtain the phase-locked solutions, case (b) above. The symmetry of the problem and the results of numerical simulations suggest one should search for solutions where the time interval between hits is fixed, say $\Delta$. Assuming an initial time $t_{0}$ at one hit, the continuity of the trajectories requires

$$
\begin{aligned}
& h_{j}\left(t_{0}+r \Delta\right) \\
& \quad=\left(\vec{e}^{(j)} \cdot \vec{s}^{(r)}\right)+\left[h_{j}\left(t_{0}+(r-1) \Delta\right)-\left(\vec{e}^{(j)} \cdot \vec{s}^{(r)}\right)\right] e^{-\Delta / \tau_{j}},
\end{aligned}
$$

with $r=1,2$, or 3 . 
By assuming that the system has a period $T=6 \Delta$, one obtains

$$
\begin{aligned}
& h_{j}\left(t_{0}\right)\left(1+e^{-3 \Delta / \tau_{j}}\right) \\
& =-\left(1-e^{-\Delta / \tau_{j}}\right) \sum_{r=1}^{3}\left(\vec{e}^{(j)} \cdot \vec{s}^{(r)}\right) e^{-(3-r) \Delta / \tau_{j}} .
\end{aligned}
$$

We choose $t_{0}$ when $x_{1}\left(t_{0}\right)=-\lambda / 2+\xi$, particle 2 is also moving toward $\lambda / 2$, and particle 3 is moving in the opposite direction. That is, we consider the sequence $\left\{\vec{s}^{(j)}\right\}$, with $j=$ $1, \ldots, 6, \vec{s}^{(j+3)}=-\vec{s}^{(j)}$, and $\vec{s}^{(1)}=\vec{s}^{(7)}$ :

$$
\begin{aligned}
\frac{2}{\lambda} \vec{s}^{(1)}= & \left(\begin{array}{r}
1 \\
1 \\
-1
\end{array}\right) \rightarrow\left(\begin{array}{r}
1 \\
-1 \\
-1
\end{array}\right) \rightarrow\left(\begin{array}{r}
1 \\
-1 \\
1
\end{array}\right) \rightarrow \\
& \rightarrow\left(\begin{array}{r}
-1 \\
-1 \\
1
\end{array}\right) \rightarrow\left(\begin{array}{r}
-1 \\
1 \\
1
\end{array}\right) \rightarrow\left(\begin{array}{r}
-1 \\
1 \\
-1
\end{array}\right)=\frac{2}{\lambda} \vec{s}^{(6)} .
\end{aligned}
$$

The initial conditions of this periodic solution are then

$$
\begin{aligned}
& h_{1}\left(t_{0}\right)\left(1+e^{-3 \Delta / \tau_{1}}\right) \\
& =-\left(1-e^{-\Delta / \tau_{1}}\right) \frac{\lambda}{2 \sqrt{3}}\left(e^{-2 \Delta / \tau_{1}}-e^{-\Delta / \tau_{1}}+1\right), \\
& h_{2}\left(t_{0}\right)\left(1+e^{-3 \Delta / \tau_{2}}\right) \\
& =-\left(1-e^{-\Delta / \tau_{2}}\right) \frac{\lambda}{\sqrt{2}}\left(e^{-2 \Delta / \tau_{2}}-1\right), \\
& h_{3}\left(t_{0}\right)\left(1+e^{-3 \Delta / \tau_{2}}\right) \\
& =\left(1-e^{-\Delta / \tau_{2}}\right) \frac{\lambda}{\sqrt{6}}\left(e^{-2 \Delta / \tau_{2}}+2 e^{-\Delta / \tau_{2}}+1\right) .
\end{aligned}
$$

Finally by inserting the initial condition $x_{1}\left(t_{0}\right)=$ $\frac{1}{\sqrt{3}} h_{1}\left(t_{0}\right)-\frac{2}{\sqrt{6}} h_{3}\left(t_{0}\right)=-\frac{\lambda}{2}+\xi$, we find the equation that fixes $\Delta$ in terms of the parameters of the problem. This is described in the Appendix. Figure 3(a) shows the trajectories of particles 1, 2, and 3 as a function of time, for one period $T=6 \Delta$. Time is expressed in units of $\tau_{0}$. Figure $3(\mathrm{~b})$ plots

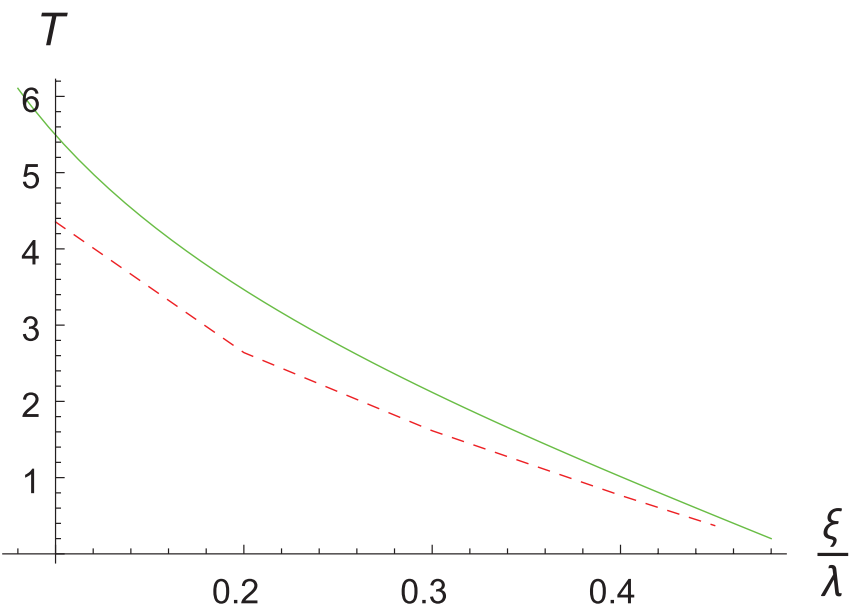

FIG. 4. (Color online) The period of the phase-locking solution (dashed line) and the period of the solution with the three oscillators in-phase (solid line) are plotted versus $\xi / \lambda$. The periods, in units of $\tau_{0}$, were evaluated with $3 a /(8 R)=\sqrt{3} / 5$, that is, $\tau_{0} / \tau_{1}=0.8$. the normal modes $h_{1}(t), h_{2}(t)$, and $h_{3}(t)$, again for one period. The oscillations of the pair of normal modes corresponding to the degenerate eigenvalue have an amplitude much larger than that of the oscillations of the normal mode $h_{1}(t)$. We verified that there exists a completely analogous phase-locked synchronized solution where the role of particles 2 and 3 are exchanged.

In Figure 4, we compare the period of the phase-locking solution with the period of the solution with the three oscillators in-phase, as a function of $\xi / \lambda$. One sees that the solution where the three particles are synchronized in-phase always has a longer period than the period of the pair of phaselocked solutions, independently of the geometric conditions. This is also the solution which appears in the experiment and in the numerical simulations [4].

\section{B. Case of $n=4$}

For $n=4$ the lowest eigenvalue of the matrix $C_{4}$ is a singlet, $\lambda_{1}=-1-\sqrt{2}$; the next is also a singlet, $\lambda_{2}=-1+\sqrt{2}$; and the next two are a doublet, $\lambda_{3}=\lambda_{4}=1$. The eigenvectors may be written as

$$
\begin{aligned}
& \vec{e}^{(1)}=\frac{1}{2}\left(\begin{array}{r}
1 \\
-1 \\
1 \\
-1
\end{array}\right), \quad \vec{e}^{(2)}=\frac{1}{2}\left(\begin{array}{l}
1 \\
1 \\
1 \\
1
\end{array}\right), \\
& \vec{e}^{(3)}=\frac{1}{\sqrt{2}}\left(\begin{array}{r}
0 \\
-1 \\
0 \\
1
\end{array}\right), \quad \vec{e}^{(4)}=\frac{1}{\sqrt{2}}\left(\begin{array}{r}
-1 \\
0 \\
1 \\
0
\end{array}\right) .
\end{aligned}
$$

The periodic solutions of the system in order of decreasing periods are

(a) adjacent particles in antiphase configuration;

(b) all particles synchronized in-phase;

(c) the pair $(1,2)$ are antiphase with the pair $(3,4)$ [also an equivalent solution where the pair $(1,4)$ are antiphase with the pair (2,3)];

(d) a pair of phase-locked solutions, with the same period.

In the antiphase configuration (a), the vector $\vec{s}$ of the positions of the potentials is proportional to the eigenvector $\vec{e}^{(1)}$. Then, all the normal modes $h_{j}(t)$ with $j=2$, 3 , or 4 obey homogeneous differential equations and may consistently vanish at all times. The normal mode $h_{1}(t)$ generates the following solution:

$$
\begin{gathered}
\vec{s}= \pm \frac{\lambda}{2}\left(\begin{array}{r}
1 \\
-1 \\
1 \\
-1
\end{array}\right), \quad\left(\vec{e}^{(1)} \cdot \vec{s}\right)= \pm \lambda, \\
h_{1}(t)=\left(\vec{e}^{(1)} \cdot \vec{x}(t)\right)=2 x_{1}(t), \\
\vec{x}_{1}(t)=-\vec{x}_{2}(t)=\vec{x}_{3}(t)=-\vec{x}_{4}(t), \\
h_{1}(t)-\left(\vec{e}^{(1)} \cdot \vec{s}\right)+\tau_{1} \frac{d}{d t} h_{1}(t)=0, \\
\text { with } \tau_{1}=\tau_{0}\left(1-\frac{(1+\sqrt{2}) 3 a}{8 R}\right)^{-1} .
\end{gathered}
$$

Here the four particles perform antiphase oscillations, satisfying the same equation as un-coupled oscillators, but with $\tau_{1}$ 
replacing $\tau_{0}$. The period of this antiphase solution is

$$
T_{1}=2 \tau_{1} \log \frac{\lambda-\xi}{\xi} \text {. }
$$

The trajectories for this case are shown in Fig. 5(a).

The next solution, case (b) above, is the in-phase synchronization and may still be written in terms of just one normal mode, $h_{2}(t)$. Its relaxation time and period are

$$
\begin{aligned}
\tau_{2} & =\tau_{0}\left(1+\frac{(\sqrt{2}-1) 3 a}{8 R}\right)^{-1}, \\
T_{2} & =2 \tau_{2} \log \frac{\lambda-\xi}{\xi}<T_{1} .
\end{aligned}
$$

In the next solution, case (c), the couple $x_{1}(t)=x_{2}(t)$ are in antiphase with the couple $x_{3}(t)=x_{4}(t)$. The vector $\vec{s}$ of the position of the potential is proportional to the sum $\vec{e}^{(3)}+\vec{e}^{(4)}$.
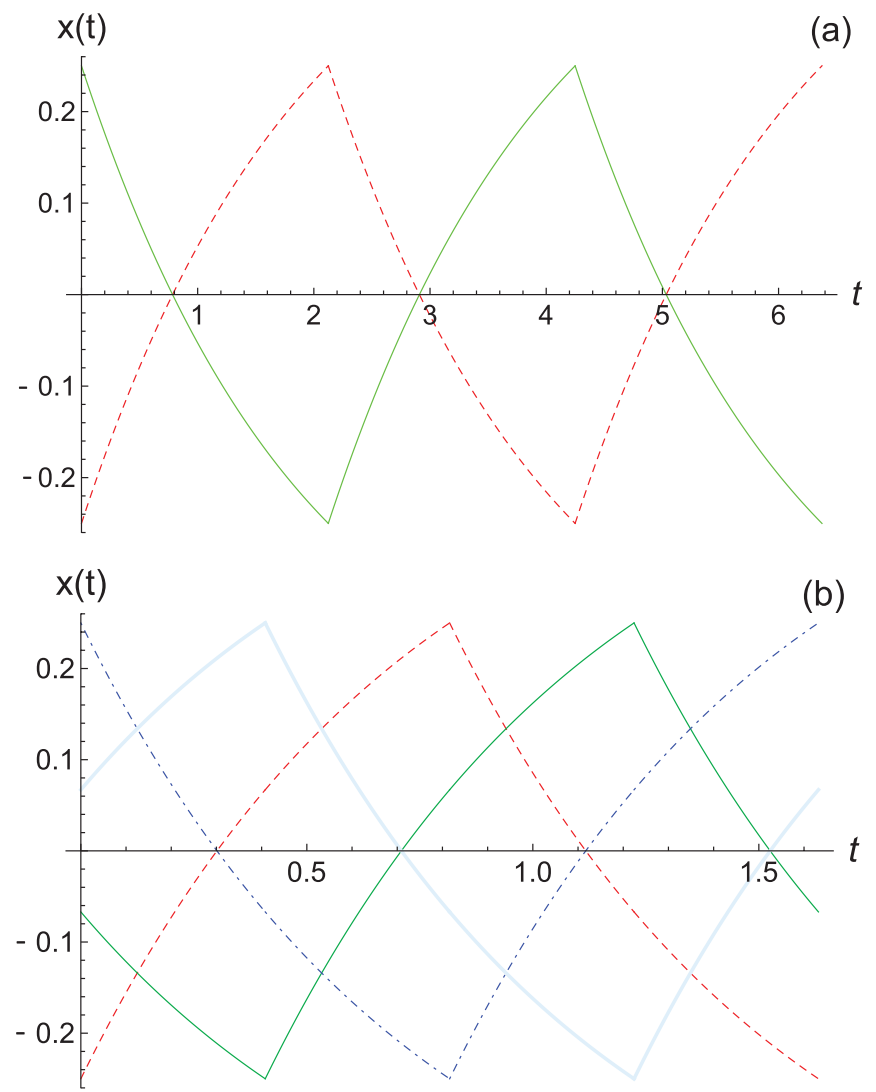

FIG. 5. (Color online) (a) The "slowest" solution (longest period) for the case $n=4$ has nearest neighbors in antiphase with each other (hence next-nearest neighbors are in-phase). The trajectories of the pair of particles 1 and 3 (red dashed line) and the other pair (green solid line) are plotted versus time, in units of $\tau_{0}$. (b) There are two equally "fastest" solutions for $n=4$. One has nearest-neighbor pairs in-phase and the other has pairs in antiphase with each other. This would look like panel (a), but with particles 1 and 2 in red and particles 3 and 4 in blue. The other solution is shown in panel (b) and has trajectories of the particles 1 to 4 , respectively, red dashed line, green solid line, blue dash-dotted line, and cyan thick line, plotted versus time, in units of $\tau_{0}$.
Then we may choose $h_{1}(t)=0, h_{2}(t)=0$, and

$$
\begin{aligned}
\vec{s} & = \pm \frac{\lambda}{2}\left(\begin{array}{c}
1 \\
1 \\
-1 \\
-1
\end{array}\right), \quad\left(\vec{e}^{(3)} \cdot \vec{s}\right)=\left(\vec{e}^{(4)} \cdot \vec{s}\right)=\mp \frac{\lambda}{\sqrt{2}}, \\
\vec{x}_{1}(t) & =\vec{x}_{2}(t)=-\vec{x}_{3}(t)=-\vec{x}_{4}(t)=-\frac{1}{\sqrt{2}} h_{4}(t), \\
h_{3}(t) & =h_{4}(t), \\
\tau_{3} & =\tau_{0}\left(1+\frac{3 a}{8 R}\right)^{-1}, \quad T_{3}=2 \tau_{3} \log \frac{\lambda-\xi}{\xi}<T_{2} .
\end{aligned}
$$

The trajectories perform oscillations as depicted in Fig. 5(a), but the relaxation time is replaced by $\tau_{3}$ and the pair of particles 1 and 2 in red and the other pair in blue. A completely equivalent solution has the pair of particles $x_{1}(t)=x_{4}(t)$ in antiphase with the pair $x_{2}(t)=x_{3}(t)$.

Finally there are phase-locked solutions, case (d) above, also with short periods $T$, where $x_{4}(t)=x_{1}(t-3 T / 4)$, $x_{3}(t)=x_{1}(t-T / 2)$, and $x_{2}(t)=x_{1}(t-T / 4)$. A completely equivalent periodic solution has the reverse order $x_{4}(t)=$ $x_{1}(t+3 T / 4), x_{3}(t)=x_{1}(t+T / 2)$, and $x_{2}(t)=x_{1}(t+T / 4)$.

Since the sequence of the positions of the potentials is $\vec{s}=$ $\frac{\lambda}{2}(1,-1,-1,1) \rightarrow-\vec{s} \rightarrow \vec{s} \rightarrow-\vec{s} \rightarrow \ldots$, we find $h_{1}(t)=$ $0, h_{2}(t)=0$, and

$$
\begin{aligned}
h_{3}\left(t_{0}\right) & =\frac{\lambda}{\sqrt{2}} \frac{\left(1-e^{-\Delta / \tau_{3}}\right)^{2}}{1+e^{-2 \Delta / \tau_{3}}}, \\
h_{4}\left(t_{0}\right) & =\frac{\lambda}{\sqrt{2}} \frac{1-e^{-2 \Delta / \tau_{3}}}{1+e^{-2 \Delta / \tau_{3}}}, \\
\text { with } \tau_{3} & =\frac{\tau_{o}}{1+\frac{3 a}{8 R}} .
\end{aligned}
$$

The period is

$$
T=4 \Delta=2 \tau_{3} \log \frac{\lambda-\xi}{\xi}=T_{3},
$$

where $\xi=\lambda \frac{e^{-2 \Delta / \tau_{3}}}{1+e^{-2 \Delta / \tau_{3}}}$,

same period as case (c), and the motion is given by

$$
\begin{aligned}
& x_{1}(t)=-\frac{1}{\sqrt{2}} h_{4}(t), \quad x_{2}(t)=-\frac{1}{\sqrt{2}} h_{3}(t), \\
& x_{3}(t)=\frac{1}{\sqrt{2}} h_{4}(t), \quad x_{4}(t)=\frac{1}{\sqrt{2}} h_{3}(t) .
\end{aligned}
$$

The trajectories for this are shown in Fig. 5(b), for particles 1 to 4 .

\section{Case of $n=5$}

The odd-numbered systems, except $n=3$ discussed above, have general properties. For $n=5$ the lowest eigenvalue of the matrix $C_{5}$ is a doublet, $\lambda_{1}=\lambda_{2}$; the next is a singlet, $\lambda_{3}$, followed by a doublet, $\lambda_{4}=\lambda_{5}$. The eigenvectors may be written as

$$
\lambda_{1}=\lambda_{2}=-\frac{1}{2} \sqrt{29+\frac{22}{\sqrt{5}}} \simeq-3.116,
$$




$$
\begin{aligned}
\vec{e}^{(1)} & =\sqrt{\frac{2}{5}}\left(\begin{array}{c}
1 \\
\cos \frac{4 \pi}{5} \\
\cos \frac{2 \pi}{5} \\
\cos \frac{2 \pi}{5} \\
\cos \frac{4 \pi}{5}
\end{array}\right), \quad \vec{e}^{(2)}=\sqrt{\frac{2}{5}}\left(\begin{array}{c}
0 \\
\sin \frac{4 \pi}{5} \\
-\sin \frac{2 \pi}{5} \\
\sin \frac{2 \pi}{5} \\
-\sin \frac{4 \pi}{5}
\end{array}\right), \\
\lambda_{3} & =\sqrt{13-\frac{22}{\sqrt{5}}} \simeq 1.778, \\
\vec{e}^{(3)} & =\sqrt{\frac{1}{5}}\left(\begin{array}{l}
1 \\
1 \\
1 \\
1 \\
1
\end{array}\right), \quad \lambda_{4}=\lambda_{5} \simeq 2.227, \\
\vec{e}^{(4)} & =\sqrt{\frac{2}{5}}\left(\begin{array}{c}
1 \\
\cos \frac{2 \pi}{5} \\
\cos \frac{4 \pi}{5} \\
\cos \frac{4 \pi}{5} \\
\cos \frac{2 \pi}{5}
\end{array}\right), \quad \vec{e}^{(5)}=\sqrt{\frac{2}{5}}\left(\begin{array}{c}
0 \\
\sin \frac{2 \pi}{5} \\
\sin \frac{4 \pi}{5} \\
-\sin \frac{4 \pi}{5} \\
-\sin \frac{2 \pi}{5}
\end{array}\right) .
\end{aligned}
$$

The periodic solutions of the system in order of decreasing periods are

(a) a pair of phase-locked solutions, one with $x_{1}(t)=x_{3}(t+2 T / 10)=x_{5}(t+4 T / 10)=x_{2}(t+6 T / 10)=$ $x_{4}(t+8 T / 10)$ and the analogous solution with opposite time-shifts;

(b) a pair of phase-locked solutions, one with $x_{1}(t)=x_{2}(t+2 T / 10)=x_{3}(t+4 T / 10)=x_{4}(t+6 T / 10)=$ $x_{5}(t+8 T / 10)$ and the analogous solution with opposite time-shifts;

(c) a synchronized in-phase solution $x_{j}(t)=x(t), j=$ $1,2, \ldots, 5$.

The periods of these solutions are shown in Fig. 6 and evaluated in the Appendix.

The phase-locked solution (a) is quite relevant since for every odd number of particles greater than 3 , the system

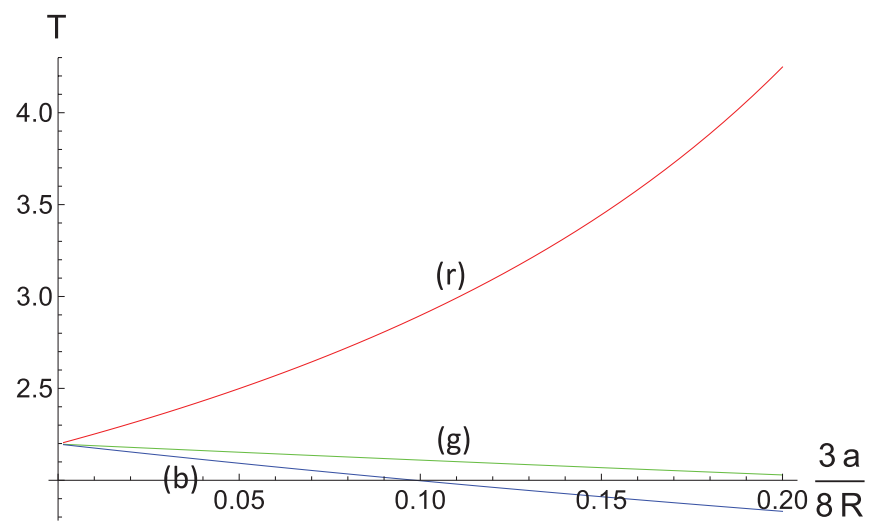

FIG. 6. (Color online) The periods for the phase-shifted solution (r) red with adjacent trajectories $\{1,3,5,2,4\}$, for the phase-shifted solution (g) green with adjacent trajectories $\{1,2,3,4,5\}$, and for the in-phase solution (b) blue, in units of $\tau_{0}$, are plotted versus the hydrodynamic coupling $3 a /(8 R)$. These periods are evaluated in the Appendix. synchronizes into a periodic solution of this type. At any time, three particles are moving in one direction and two are moving in the opposite direction. We call $\Delta$ the uniform time interval between any two consecutive hits. The period $T=10 \Delta$. Take, for example, that at time $t_{0}$ particles 1,3 , and 5 are moving toward the positive direction, with $x_{1}\left(t_{0}\right)<x_{3}\left(t_{0}\right)<x_{5}\left(t_{0}\right)$. Then the sequence of the vectors $\left\{\vec{s}^{(j)}\right\}$, with $j=1, \ldots, 10$, $\vec{s}^{(j)}=-\vec{s}^{(j+5)}$, is

$$
\begin{aligned}
\frac{2}{\lambda} \vec{s}^{(1)}= & \left(\begin{array}{r}
1 \\
-1 \\
1 \\
-1 \\
1
\end{array}\right) \rightarrow\left(\begin{array}{r}
1 \\
-1 \\
1 \\
-1 \\
-1
\end{array}\right) \rightarrow\left(\begin{array}{r}
1 \\
-1 \\
1 \\
1 \\
-1
\end{array}\right) \rightarrow\left(\begin{array}{r}
1 \\
-1 \\
-1 \\
1 \\
-1
\end{array}\right) \\
& \rightarrow\left(\begin{array}{r}
1 \\
1 \\
-1 \\
1 \\
-1
\end{array}\right) \rightarrow\left(\begin{array}{r}
-1 \\
1 \\
-1 \\
1 \\
-1
\end{array}\right)=\frac{2}{\lambda} \vec{s}^{(6)} .
\end{aligned}
$$

No eigenvector $\vec{e}^{(j)}$ is orthogonal to all of the eigenvectors $\left\{\vec{s}^{(j)}\right\}$ of this sequence, therefore all five normal modes $h_{j}(t)$ contribute to the solution.

The continuity and periodicity conditions fix the initial conditions:

$$
\begin{aligned}
& h_{j}\left(t_{0}\right)\left(1+e^{-5 \Delta / \tau_{j}}\right) \\
& =-\left(1-e^{\Delta / \tau_{j}}\right) \sum_{r=1}^{5}\left(\vec{e}^{(j)} \cdot \vec{s}^{(r)}\right) e^{-(5-r) \Delta / \tau_{j}} \\
& =-\frac{\lambda}{2} \vec{e}^{(j)} \cdot\left(\begin{array}{c}
1-e^{-5 \Delta / \tau_{j}} \\
1-2 e^{-\Delta / \tau_{j}}+e^{-5 \Delta / \tau_{j}} \\
-1+2 e^{-2 \Delta / \tau_{j}}-e^{-5 \Delta / \tau_{j}} \\
1-2 e^{-3 \Delta / \tau_{j}}+e^{-5 \Delta / \tau_{j}} \\
-1+2 e^{-4 \Delta / \tau_{j}}-e^{-5 \Delta / \tau_{j}}
\end{array}\right), \\
& \text { and as usual } \tau_{j}=\frac{\tau_{0}}{1+\frac{3 a}{8 R} \lambda_{j}} .
\end{aligned}
$$

One last equation determines the time interval $\Delta$. If we use $x_{1}\left(t_{0}\right)=-\frac{\lambda}{2}+\xi$, it is

$$
-\frac{\lambda}{2}+\xi=\sqrt{\frac{2}{5}}\left[h_{1}\left(t_{0}\right)+h_{4}\left(t_{0}\right)\right]+\sqrt{\frac{1}{5}} h_{3}\left(t_{0}\right) .
$$

The trajectories are shown in Fig. 7(a), for particles 1 to 5 (the coupling is chosen at $3 a /(8 R)=0.2$, and then the period of the solution is $\left.T \simeq 3.8547 \tau_{0}\right)$.

Figure 7(b) shows the complex behavior of normal modes $h_{j}(t), j=1,2, \ldots 5$. The doublet of normal modes 1 and 2 presents oscillations with an amplitude much larger than that of the oscillations of the doublet of normal modes 4 and 5 . The singlet normal mode 3 , related to the center of mass $\sum_{j=1}^{5} x_{j}(t)$, has even smaller oscillations.

Another pair of phase-locked solutions, case (b), with a shorter period has the trajectories of particles $\{1,2,3,4,5\}$ and the equivalent solution with the arrangement of trajectories $\{1,5,4,3,2\}$. Here, like in case (a), at any time there are three particles moving in one direction and two moving in 

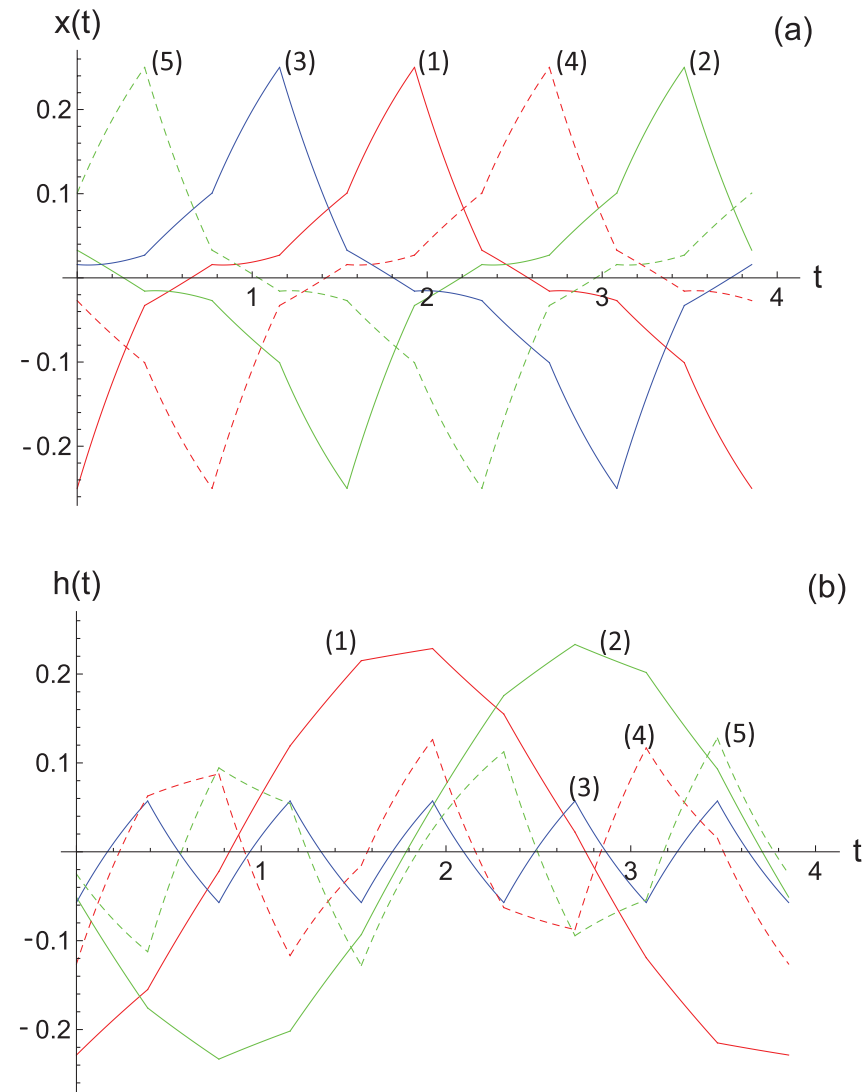

FIG. 7. (Color online) This phase-locked solution for $n=5$, with the nearest neighbors almost in antiphase, is the solution with the longest period and is the stable state with attractive potentials. (a) The trajectories of particles 1 to 5, respectively, (1), (2)...(5) red solid line, green solid line, blue solid line, red dashed line, and green dashed line, are plotted versus time, in units of $\tau_{0}$. To draw the figure, the coupling was chosen as $3 a /(8 R)=0.2$, and then the period of the solution is $T \simeq 3.8547 \tau_{0}$. (b) The normal modes $h_{j}(t), j=1,2, \ldots 5$, respectively, (1), (2)...(5) red solid line, green solid line, blue solid line, red dashed line, and green dashed line, are plotted versus time, in units of $\tau_{0}$.

the opposite direction. We call $\Delta$ the uniform time interval between two hits. The period $T=10 \Delta$. Taking, for example, that at time $t_{0}$ the particles 1,4 , and 5 are moving toward the positive direction, with $x_{1}\left(t_{0}\right)<x_{5}\left(t_{0}\right)<x_{4}\left(t_{0}\right)$, then the sequence of the vectors $\left\{\vec{s}^{(j)}\right\}$, with $j=1, \cdots, 10, \vec{s}^{(j)}=$ $-\vec{s}^{(j+5)}$, is

$$
\begin{aligned}
\frac{2}{\lambda} \vec{s}^{(1)}= & \left(\begin{array}{r}
1 \\
-1 \\
-1 \\
1 \\
1
\end{array}\right) \rightarrow\left(\begin{array}{r}
1 \\
-1 \\
-1 \\
-1 \\
1
\end{array}\right) \rightarrow\left(\begin{array}{r}
1 \\
1 \\
-1 \\
-1 \\
1
\end{array}\right) \rightarrow \\
& \rightarrow\left(\begin{array}{r}
1 \\
1 \\
-1 \\
-1 \\
-1
\end{array}\right) \rightarrow\left(\begin{array}{r}
1 \\
1 \\
1 \\
-1 \\
-1
\end{array}\right) \rightarrow\left(\begin{array}{r}
-1 \\
1 \\
1 \\
-1 \\
-1
\end{array}\right)=\frac{2}{\lambda} \vec{s}^{(6)} .
\end{aligned}
$$

As before, the continuity and periodicity conditions fix the initial conditions:

$$
\begin{aligned}
& h_{j}\left(t_{0}\right)\left(1+e^{-5 \Delta / \tau_{j}}\right) \\
& =-\left(1-e^{\Delta / \tau_{j}}\right) \sum_{r=1}^{5}\left(\vec{e}^{(j)} \cdot \vec{s}^{(r)}\right) e^{-(5-r) \Delta / \tau_{j}} \\
& =-\frac{\lambda}{2} \vec{e}^{(j)} \cdot\left(\begin{array}{c}
1-e^{-5 \Delta / \tau_{j}} \\
1-2 e^{-3 \Delta / \tau_{j}}+e^{-5 \Delta / \tau_{j}} \\
1-2 e^{-\Delta / \tau_{j}}+e^{-5 \Delta / \tau_{j}} \\
-1+2 e^{-4 \Delta / \tau_{j}}-e^{-5 \Delta / \tau_{j}} \\
-1+2 e^{-2 \Delta / \tau_{j}}-e^{-5 \Delta / \tau_{j}}
\end{array}\right), \\
& \text { and as usual } \tau_{j}=\frac{\tau_{0}}{1+\frac{3 a}{8 R} \lambda_{j}} .
\end{aligned}
$$

By using the same initial condition, $x_{1}\left(t_{0}\right)=-\frac{\lambda}{2}+\xi$, the equation that determines $\Delta$ still has the form of Eq. (15). The trajectories are shown in Fig. 8(a), for particles 1 to 5. In
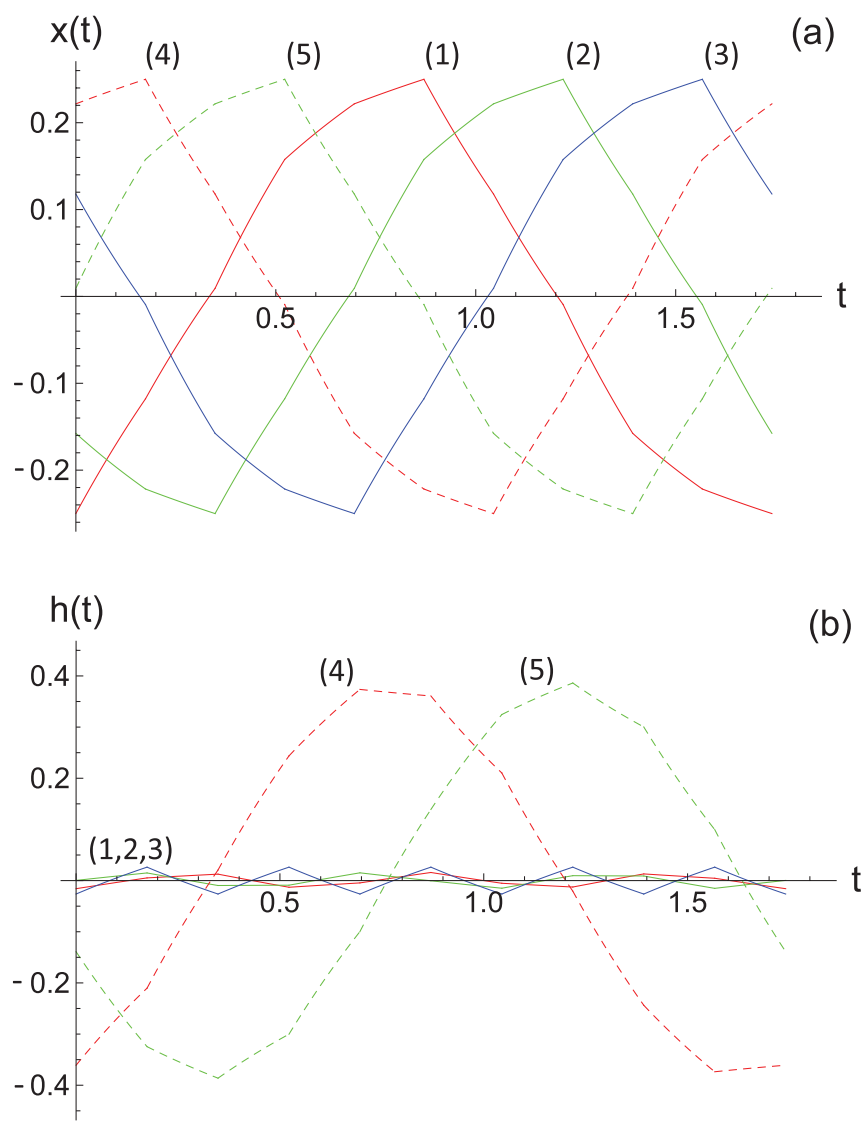

FIG. 8. (Color online) This phase-locked solution has a shorter period than the one depicted in Fig 7. (a) The trajectories of particles 1 to 5 , respectively, (1), (2) ... (5) red solid line, green solid line, blue solid line, red dashed line, and green dashed line, are plotted versus time, in units of $\tau_{0}$. To draw the figure, the coupling $3 a /(8 R)=0.2$, then the period of the solution is $T \sim 1.7407 \tau_{0}$. (b) The normal modes $h_{j}(t), j=1,2, \ldots 5$, respectively, (1), (2) ...(5) red solid line, green solid line, blue solid line, red dashed line, and green dashed line, are plotted versus time, in units of $\tau_{0}$. To draw the figure, the coupling $3 a /(8 R)=0.2$. 
the figure, the coupling is $3 a /(8 R)=0.2$, and then the period of this solution is $T \sim 1.7407 \tau_{0}$.

Figure 8(b) shows the normal modes $h_{j}(t), j=1,2, \ldots 5$. The doublet of normal modes 4 and 5, which has the shortest relaxation time, oscillates with much larger amplitudes than either the doublet of normal modes 1 and 2 or the singlet normal mode 3 .

The synchronized in-phase solution, case (c), for the five particles is very simple, since only the mode $h_{3}(t)$ contributes, whilst all the $h_{j}(t)=0$ for $j \neq 3$. The relaxation time and period are

$$
\tau_{3}=\tau_{0}\left(1+\frac{3 a}{8 R} \lambda_{3}\right)^{-1}, \quad T_{3}=2 \tau_{3} \log \frac{\lambda-\xi}{\xi} .
$$

This is the "fastest" periodic solution.

\section{Case of higher $n$}

As the number of particles of the system increases, the analytic periodic solutions of the system become more and more complex. Yet a few simple features are generic.

We already mentioned that for every particle number $n$ being an even integer, the lowest eigenvalue $\lambda_{1}$ is a singlet, its eigenvector is $\vec{e}^{(1)}=\frac{1}{\sqrt{n}}(1,-1,1, \ldots,-1)$. This allows a periodic solution where adjacent particles are in antiphase, just like the first solution (a) described for the system of four particles. Since $h_{1}(t)$ is the unique normal mode nonvanishing, the period of the antiphase solution is

$$
T_{1}=2 \tau_{1} \log \frac{\lambda-\xi}{\xi}, \quad \tau_{1}=\tau_{0}\left(1+\frac{3 a}{8 R} \lambda_{1}\right)^{-1},
$$

where

$$
\lambda_{1}=(-1)^{n / 2+1}-2 \sum_{j=1}^{n / 2-1}(-1)^{j} \frac{\cos \frac{2 j \pi}{n}+\cos ^{2} \frac{j \pi}{n}}{\sin \frac{j \pi}{n}},
$$

that is, in particular,

$$
\begin{aligned}
& \lambda_{1} \sim-2.414(n=4), \\
& \lambda_{1} \sim-4.577(n=6), \\
& \lambda_{1} \sim-6.528(n=8) .
\end{aligned}
$$

This antiphase solution is the periodic solution with the longest period, for every even $n$.

We also mentioned that for every $n$ being an odd integer $n \geqslant 5$, the lowest eigenvalue $\lambda_{1}$ is a doublet. The periodic solution with the longest period is analogous to the solution (a) for the system of five particles. That is a pair of equivalent solutions where a sequence of trajectories are time shifted by a uniform time interval. For $n=7$ the pair of sequences is $\{1,3,5,7,2,4,6\}$ and $\{1,6,4,2,7,5,3\}$. One may notice that if $n>>1$, adjacent particles are time shifted by almost a half period; that is, they are approximately in antiphase. Thus the distinction between even and odd number $n$ decreases, as $n$ increases.

\section{E. Simulations}

To verify which of the analytical solutions is the dynamical steady state, we resorted to numerical integration. Two different codes have been developed.
First, we solved the coupled system of Eq. (2) which corresponds to the tangential motion, using the solver ODE113 of MATLAB, which implements the Adams-Bashforth-Moulton multistep (i.e., timestep optimizing) method. We used the event handling facility integrated into all MATLAB's ordinary differential equation (ODE) solvers to intercept the instant of time at which any one of the particles hits the switch position. As a stabilizing measure, we imposed that at a "hit" all particles which are in the range of $\delta s$ from their switches are considered to have hit their target and consequently their centers of force switch their positions; several values in the interval $10^{-8} \lambda \leqslant \delta s \leqslant 10^{-5} \lambda$ have been used with no substantial difference in the results.

Second, a different code described in Refs. $[3,4,14]$ with a fixed timestep, is also used, enabling testing in the absence of noise but also the robustness at finite temperature. As well as exploring noise, this Brownian dynamics (BD) code solves a model which is very close to the experimental condition [4]: the colloidal particles are free to move in a two-dimensional environment (for which the description in terms of $n$ rather than $2 n$ normal modes is an approximation), and the tangential and normal forces can be set independently at different stiffnesses. Finally, experiments typically work at a finite sampling rate, and this can be mimicked closely in the fixed-timestep BD code as was discussed in a previous study [3].

The numerical solution (both the simulation approaches described above) show the remarkable result that for every particle number $n$ and every initial condition, the system evolves to a synchronized configuration corresponding to the periodic solution with the longest period (for the case of positive trap stiffness $\kappa>0$ discussed up to here). If such a configuration is a pair of equivalent solutions, as it happens for odd $n \geqslant 5$, the synchronization occurs on either one of the pair. These findings also agree with the experiment in Ref. [4].

Another result of the extensive numerical solutions is that we never observe solutions different from the cases that were investigated analytically (in principle, we could not have ruled out the presence of much more complex and less symmetric solutions).

\section{REPULSIVE POTENTIAL}

It is interesting to consider a harmonic repulsive potential, $\kappa<0$, acting on the colloidal spheres. This may be realized experimentally by tailoring an appropriate potential landscape with time-sharing or holographical optical traps. Then the synchronization of the system of oscillating particles due to the hydrodynamical interaction still occurs, but on a periodic configuration different from the one observed in the attractive case. With minimal changes from the previous analysis we can describe the periodic solutions occurring in the case of repulsive harmonic potentials. Again the numerical simulations of this case confirm that the systems converge onto one of the analytical solutions, and in this case it is one with a short period.

With negative stiffness, if we ignore the hydrodynamic coupling, a particle moves toward the positive $x$ axis according to the differential equation $\gamma_{0} \dot{x}(t)+\kappa\left[x(t)+\frac{\lambda}{2}\right]-f(x)=0$, where now $\kappa<0$ is the stiffness of the repulsive harmonic force, $\tau_{0}=\gamma_{0} /|\kappa|$, and $f$ is a stochastic force due to thermal agitation of the fluid, which will be ignored in the analysis 


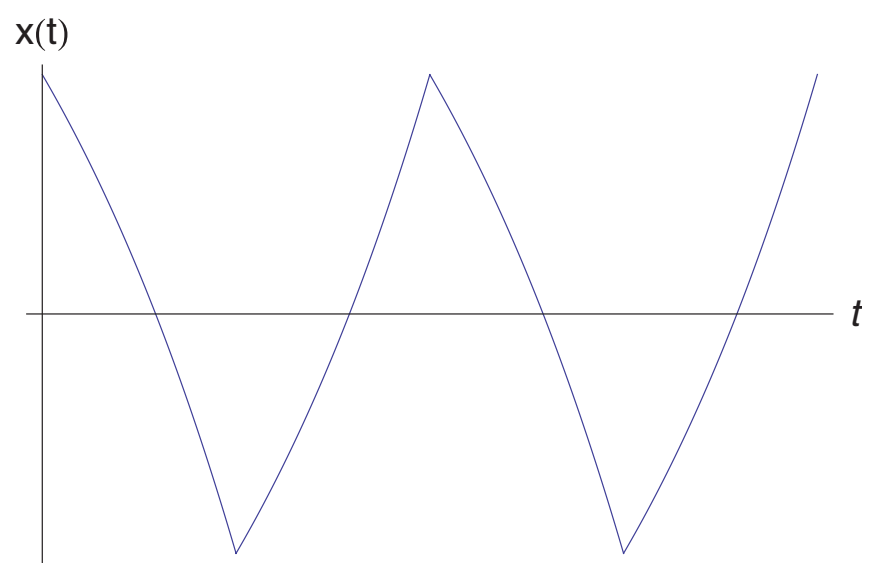

FIG. 9. (Color online) Trajectory of a driven oscillator with repulsive driving force, uncoupled to other oscillators. Note the increase in velocity between switch positions, characteristic of the motion with potentials of negative stiffness.

of the deterministic system. In this case, the motion is exponentially accelerated until the particle reaches the position $\frac{\lambda}{2}-\xi$. At that time the center of the repulsive harmonic potential moves from the position $-\frac{\lambda}{2}$ to $\frac{\lambda}{2}$. The particle inverts its motion until it reaches the position $-\frac{\lambda}{2}+\xi$ when the potential jumps again. The resulting oscillatory motion for a single particle (or one in a noncoupled system), neglecting the stochastic force, is depicted in Fig. 9.

The period $T_{0}$ of these uncoupled oscillations is $T_{0}=$ $2 \tau_{0} \log \frac{\lambda-\xi}{\xi}$. The oscillatory motion is similar to the attractive potential case, shown in Fig. 1, except for the concavity.

One may repeat the analysis of the attractive case and obtain the linear deterministic system, valid in a time interval between two hits:

$$
\left(I+\frac{3 a}{8 R} C_{n}\right)[\vec{x}(t)-\vec{s}]-\tau_{0} \frac{d}{d t} \vec{x}(t)=0, \tau_{0}=\frac{\gamma_{0}}{|\kappa|},
$$

where $\vec{x}(t)$ is the $n$-component vector of the (local) position of the particles, $\vec{s}$ is the $n$ component vector of the (local) position of the minima of the repulsive potential proper for such a time interval (opposite of the previous case), and $C_{n}$ is the same real symmetric circulant matrix of order $n$ given in Eq. (3). We again define the normal modes $h_{j}(t)=\left[\vec{e}^{(j)}, \vec{x}(t)\right]$ and their decoupled equations:

$$
h_{j}(t)-\left(\vec{e}^{(j)} \cdot \vec{s}\right)-\tau_{j} \frac{d}{d t} h_{j}(t)=0, \tau_{j}=\frac{\tau_{0}}{1+\frac{3 a}{8 R} \lambda_{j}} .
$$

They differ from the previous Eqs. (5) only for the sign of the time evolution. It follows that the set of periodic solutions, described in the previous analysis for the attractive traps, still exists in the present case of repulsive traps, with the same periods. Only the shape of the trajectories is affected by replacing diverging exponentials in place of converging ones.

Figures 10(a) and 10(b) are examples of this point, when compared with the Figs. 3(a) and 3(b). We plotted the phase-locked solution for three particles in the repulsive case. Figure 10(a) shows the trajectories of the three particles as a function of time, for one period $T=6 \Delta$. Figure $10(\mathrm{~b})$ plots the normal modes $h_{1}(t), h_{2}(t)$, and $h_{3}(t)$, again for one period.
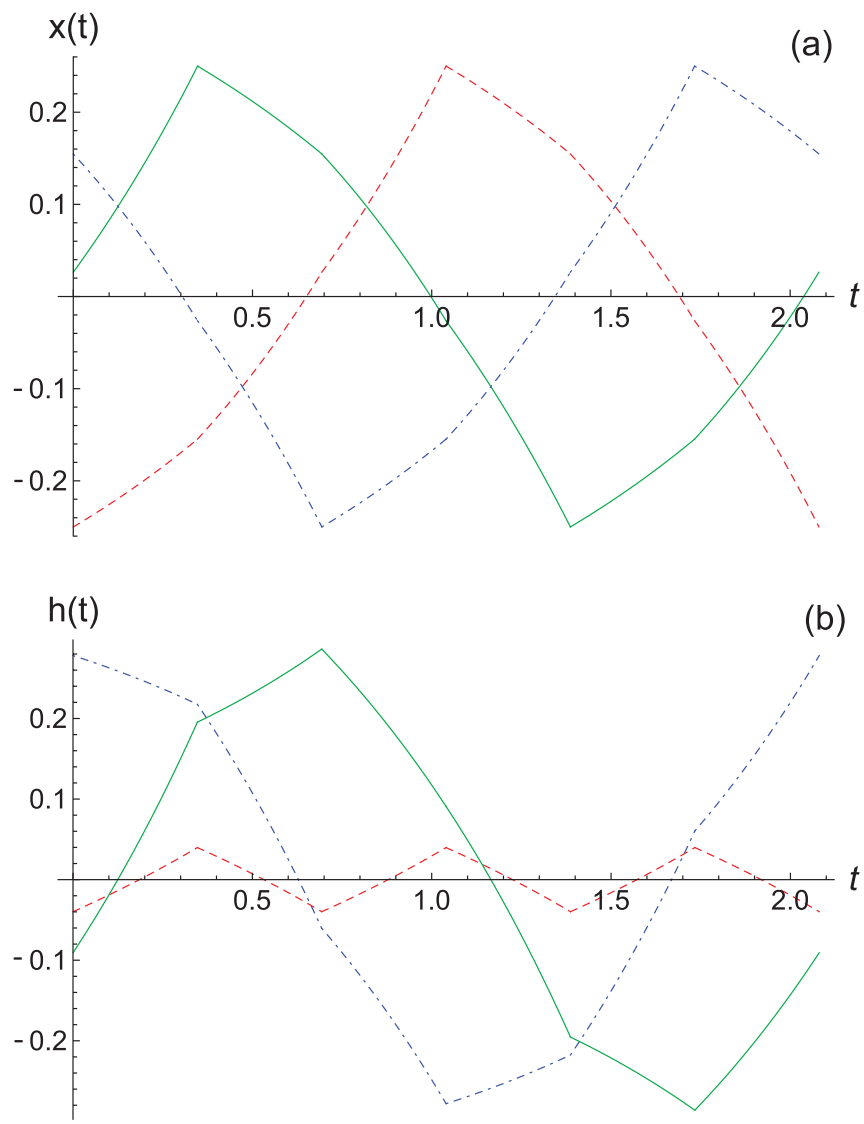

FIG. 10. (Color online) (a) The trajectories for the the phaselocked solution for three particles in the repulsive case are plotted versus time, in units of $\tau_{0}$. (b) The normal modes $h_{1}(t)$ (red dashed line), $h_{2}(t)$ (green solid line), and $h_{3}(t)$ (blue dash-dotted line) are plotted versus time, in units of $\tau_{0}$.

The oscillations of the pair of normal modes corresponding to the degenerate eigenvalue have an amplitude much bigger than that of the oscillations of the normal mode $h_{1}(t)$. Remarkably, the simulations show that in the case of repulsive traps it is this periodic solution, the one with the shortest period, which is the stable asymptotic synchronization for the system.

With systems of higher $n$, we have observed in the numerical simulations that again upon switching from attractive to repulsive traps the stable solution ceases to be that with the longest period. Instead, the system converges onto one of the solutions with the shortest period. However, as is clear by observing the sequence of solution periods $T$ calculated for the cases ( $n=4$ or $n=5$ ), while there is a clearly separate largest $T$, there are various solutions with small $T$. We have tested numerical solutions in the physically realistic parameter space, using the two numerical approaches outlined in Sec. IV E: the ODE solver with the "strong" tangential motion constraint converges to the shortest period solution, whereas the BD simulation with the weaker "harmonic" constraint converges to one of the shorter period solutions, but not always the shortest one. This will be explored further in future work; our current understanding is that the system with repulsive potentials converges to one of the solutions with shorter periods and that 
when there are various periods grouped close together the details of the system play a strong role in selecting between these.

\section{CONCLUSION}

The model studied in this paper has several unusual features. Most studies on synchronization show that it is a threshold process: it occurs when the coupling among the oscillators is strong enough or when increasing the number of oscillators, a large fraction synchronize to a common frequency [15]. In contrast, in the present model, where we studied the deterministic system without stochastic forces, we find no threshold. Synchronization occurs for any strength of the hydrodynamic coupling, that is, any value of $a / R$, and any number of oscillators.

Most models on synchronization are described by nonlinear differential equations where a very limited progress is achieved analytically, whereas the present model is piecewise linear and all periodic solutions may be derived.

Numerical solutions in the case of attractive harmonic potentials clearly show the synchronization to the periodic solution with the longest period, for any number of particles. In the case of repulsive harmonic potentials, numerical simulations show the synchronization to the pair of phase-locked solutions in the case of three particles. We have not performed a stability analysis about the periodic solutions, but there is no reason to expect disagreement with the results of numerical simulations. This change of the asymptotic solution with the change of curvature of the potential had already been shown numerically for a linear chain in Ref. [12]. The analytical framework developed here adequately describes experiments on systems of colloidal particles with moving optical traps [4].

\section{ACKNOWLEDGMENTS}

We are grateful for many discussions with Filippo de Lillo, Nicolas Bruot, and Romain Lhermerout.

\section{APPENDIX}

In the case of three particles with attractive harmonic traps, the initial conditions for the normal modes for periodic phase- locked solutions are given in Eqs. (9). We supplement them with an initial condition,

$$
x_{1}\left(t_{0}\right)=\frac{1}{\sqrt{3}} h_{1}\left(t_{0}\right)-\frac{2}{\sqrt{6}} h_{3}\left(t_{0}\right)=-\frac{\lambda}{2}+\xi,
$$

and obtain an equation that determines $\Delta$ :

$$
\begin{aligned}
\frac{\lambda}{6}(1 & \left.-e^{-\Delta / \tau_{1}}\right)\left(e^{-2 \Delta / \tau_{1}}-e^{-\Delta / \tau_{1}}+1\right)\left(1+e^{-3 \Delta / \tau_{2}}\right) \\
& +\frac{\lambda}{3}\left(1-e^{-\Delta / \tau_{2}}\right)\left(e^{-2 \Delta / \tau_{2}}+2 e^{-\Delta / \tau_{2}}+1\right)\left(1+e^{-3 \Delta / \tau_{1}}\right) \\
= & \left(\frac{\lambda}{2}-\xi\right)\left(1+e^{-3 \Delta / \tau_{1}}\right)\left(1+e^{-3 \Delta / \tau_{2}}\right) .
\end{aligned}
$$

Furthermore, $\tau_{0} / \tau_{1}+2 \tau_{0} / \tau_{2}=3$, since $\lambda_{1}+2 \lambda_{2}=0$. Let us define

$$
x=e^{-\Delta / \tau_{0}}, \quad p_{1}=\frac{\tau_{0}}{\tau_{1}}<1, \quad p_{2}=\frac{\tau_{0}}{\tau_{2}}>1, \quad p_{1}+2 p_{2}=3,
$$

then Eq. (A1) may be rewritten in the simpler form:

$$
\begin{aligned}
& \frac{\lambda}{3}\left[-x^{p_{1}}+x^{2 p_{1}}-x^{3 p_{1}}+x^{p_{2}}\left(1+x^{p_{1}}-x^{2 p_{1}}+2 x^{3 p_{1}}\right)\right. \\
& \left.\quad+x^{2 p_{2}}\left(-2-x^{p_{1}}+x^{2 p_{1}}-3 x^{3 p_{1}}\right)\right] \\
& \quad+\xi\left(1+x^{3 p_{1}}\right)\left(1-x^{p_{2}}+x^{2 p_{2}}\right)=0 .
\end{aligned}
$$

If we choose the coupling strength $3 a /(8 R)=\sqrt{3} / 5$, we have $p_{1}=0.8$ and $p_{2}=1.1$. The further behavior of $\Delta$ as a function of $\lambda / \xi$ is depicted in Fig. 4.

If we further choose $\xi=\lambda / 4$, then Eq. (A1) implies $x \sim 0.70701565$, that is,

$$
\frac{\Delta}{\tau_{0}}=-\log x \sim 0.346702, T=6 \Delta=-6 \tau_{0} \log x .
$$

For the case of five particles with attractive traps, Fig. 6 shows the period $T$ in units of $\tau_{0}$ for the phase-shifted solution with adjacent trajectories $\{1,3,5,2,4\}$, the period of the phase-shifted solution with adjacent trajectories $\{1,2,3,4,5\}$, and the in-phase solution versus the hydrodynamic coupling $3 a /(8 R)$, while we fixed $\lambda=1$ and $\xi=0.25$. In the limit of vanishing coupling, the three curves converge to $T=$ $2 \tau_{0} \log (\lambda-\xi) / \xi \sim 2.1972 \tau_{0}$.
[1] S. Gueron, K. Levit-Gurevich, N. Liron, and J. Blum, Proc. Natl. Acad. Sci. USA 94, 6001 (1997).

[2] R. Golestanian, J. Yeomans, and N. Uchida, Soft Matter 7, 3074 (2011).

[3] J. Kotar, M. Leoni, B. Bassetti, M. Cosentino Lagomarsino, and P. Cicuta, Proc. Natl. Acad. Sci. USA 107, 7669 (2010).

[4] L. Damet, G. M. Cicuta, J. Kotar, M. Cosentino Lagomarsino, and P. Cicuta, e-print arXiv:1110.1526.

[5] J. Happel and H. Brenner, Low Reynolds Number Hydrodynamics: With Special Applications to Particulate Media (Kluwer, New York, 1983).

[6] R. J. Hunter, Foundations of Colloid Science (Oxford University Press, New York, 1981)

[7] E. Lauga and T. R. Powers, Rep. Prog. Phys. 72, 096601 (2009).
[8] M. Polin, D. G. Grier, and S. Quake, Phys. Rev. Lett. 96, 088101 (2006).

[9] R. DiLeonardo, S. Keen, J. Leach, C. D. Saunter, G. D. Love, G. Ruocco, and M. J. Padgett, Phys. Rev. E 76, 061402 (2007).

[10] G. M. Cicuta, J. Kotar, A. T. Brown, J. H. Noh, and P. Cicuta, Phys. Rev. E 81, 051403 (2010).

[11] M. C. Lagomarsino, P. Jona, and B. Bassetti, Phys. Rev. E 68, 021908 (2003).

[12] C. Wollin and H. Stark, Eur. Phys. J. E 34, 42 (2011).

[13] N. Uchida and R. Golestanian, Phys. Rev. Lett. 106, 058104 (2011).

[14] D. L. Ermak and J. A. McCammon, J. Chem. Phys. 69, 1352 (1978).

[15] A. Pikovsky, M. Rosenblum, and J. Kurths, Synchonization (Cambridge University Press, Cambridge, UK, 2001). 